\title{
Pancreatic adenocarcinoma up-regulated factor as a target for the treatment of serous ovarian adenocarcinoma
}

\section{Yeon Jeong Kim}

Department of Biological Sciences, Dong-A University

Jin Park

Asan Institute for Life Sciences, Asan Medical Center, University of Ulsan College of Medicine

Hyeon Hee Jeong

Department of Biological Sciences, Dong-A University

Ji Eun Baek

Department of Biological Sciences, Dong-A University

\section{Seung-Mo Hong}

Department of Pathology, Asan Medical Center, University of Ulsan College of Medicine

\section{Seong-Yun Jeong}

Asan Institute for Life Sciences, Asan Medical Center, University of Ulsan College of Medicine

\section{Sang Seok Koh ( $\nabla$ sskoh@dau.ac.kr)}

Department of Biological Sciences, Dong-A University

\section{Research Article}

Keywords: PAUF, ovarian cancer, targeted therapy, antibody, treatment

Posted Date: March 17th, 2021

DOl: https://doi.org/10.21203/rs.3.rs-301205/v1

License: (c) (i) This work is licensed under a Creative Commons Attribution 4.0 International License. Read Full License

Version of Record: A version of this preprint was published at Frontiers in Pharmacology on May 6th, 2022. See the published version at https://doi.org/10.3389/fphar.2022.890614. 


\section{Abstract}

Ovarian cancer is the deadliest gynecological cancer and when diagnosed at advanced stages the fiveyear survival rate is typically less than $30 \%$. Pancreatic adenocarcinoma up-regulated factor (PAUF) plays a crucial role in tumor progression, angiogenesis, and immune evasion in the pancreatic tumor microenvironment. The current study aimed to characterize the potential tumorigenic role of PAUF in serous ovarian cancer as high expression of the factor is correlated with poor prognosis in epithelial ovarian cancer. Extracellular treatment with recombinant PAUF activated intracellular signal pathways that subsequently led to enhanced proliferation, migration, and invasion in various serous ovarian adenocarcinoma cell lines. A PAUF-knockout OVCAR-5 cell line demonstrated an apparent reduction in tumor incidence and delayed tumor growth in mouse xenograft experiments. Furthermore, the administration of an anti-PAUF antibody exhibited a notable therapeutic efficacy in mice bearing the OVCAR-5 cell line-derived xenograft tumors. Taken together, our results suggest that PAUF plays a role in the progression of serous ovarian adenocarcinoma and may serve as a novel therapeutic target of the disease.

\section{Introduction}

Ovarian cancer is the deadliest gynecological cancer, and as so few symptoms appear in the early stages, more than $70 \%$ of patients are diagnosed at advanced stages $[1,2]$. The five-year survival rate of the patients with advanced stage ovarian cancer is less than $30 \%$. Epithelial ovarian cancer is the most common type of ovarian cancer and serous adenocarcinoma, which accounts for $75 \sim 80 \%$ of the epithelial type is an aggressive disease [3]. Current treatments for serous ovarian cancer include volumereduction surgery and platinum-based chemotherapy; the primary drugs used are cisplatin or a carboplatin and paclitaxel regimen [4]. However, the effects of these chemical drug therapies have been reported to be insignificant with poor patient outcomes due to drug resistance of ovarian cancer cells and rapid tumor recurrence $[5,6]$. Thus, there remains a need for better diagnostic tools, treatment interventions, and novel biomarkers for serous ovarian cancer.

We have previously reported that pancreatic adenocarcinoma up-regulated factor (PAUF) is a secreted metastasis factor in the pancreatic tumor microenvironment [7]. PAUF is overexpressed in pancreatic ductal adenocarcinoma (PDAC) and exerts its cellular functions in both autocrine and paracrine manners. PAUF acts on: i) pancreatic cancer cells to promote tumorigenic progression and metastasis [7-11], ii) endothelial cells to induce angiogenesis in a tumor mass [12], and iii) immune cells to evade immune surveillance in the tumor microenvironment $[13,14]$. Recent reports indicate that PAUF is overexpressed in ovarian cancer $[15,16]$, which is consistent with our previous observation that the PAUF transcript is elevated in the disease [7]. PAUF expression is associated with tumor grade and chemoresistance of epithelial ovarian cancer $[15,16]$. Furthermore, high expression of PAUF correlates with poor prognosis for both overall and progression-free survivals in epithelial ovarian cancer. However, the role of PAUF as a biomarker and/or therapeutic target in ovarian cancer remains unclear. 
The current study aimed to identify the function of PAUF in serous ovarian cancer using cell-based assays and mouse xenograft experiments. Findings indicate that PAUF is a useful biomarker and a novel therapeutic target for the treatment of serous ovarian cancer.

\section{Materials And Methods}

\section{Cell culture and reagents}

Human serous ovarian adenocarcinoma cell lines (HeyA8, A2780, SK-OV-3, OVCAR-5, OVCAR-8) were obtained from the National Cancer Center of Korea. The Lenti-XTM 293T cell line was obtained from Clontech. Cells were maintained at $37^{\circ} \mathrm{C}$ in a humidified atmosphere containing $5 \% \mathrm{CO}_{2}$ and cultured in the medium recommended by the suppliers: Roswell Park Memorial Institute (RPMI) was used for HeyA8, A2780, SK-OV-3, OVCAR-5 and OVCAR-8 and Dulbecco's modified Eagle's medium (DMEM) was used for Lenti-XTM 293T. Culture media were supplemented with 10\% fetal bovine serum (FBS), 2 mM Lglutamine, $1 \mathrm{mM}$ sodium pyruvate, $100 \mu \mathrm{g} / \mathrm{ml}$ streptomycin, and $100 \mathrm{IU} / \mathrm{ml}$ penicillin. RPMI, DMEM, and FBS were purchased from GE Healthcare Life Science. Cells were periodically observed by monitoring cell morphology and growth rates, and mycoplasma contamination was monitored using a mycoplasma detection kit (Lonza). Authentication of the cell lines was done using short tandem repeat (STR) profiling by the National Cancer Center of Korea with proper STR references. Recombinant PAUF protein was prepared as previously described [8]. Humanized anti-PAUF monoclonal antibody was prepared as described (KR Patent 10-1856904, 2018). Human immunoglobulin G (Thermo Scientific) was used as a control for IgG. Antibodies against ERK, p-ERK, Src, p-Src, AKT, p-AKT and Cas9 were obtained from Cell Signaling Technology. Anti- $\beta$-actin antibody was obtained from Santa Cruz Biotechnology.

\section{Generation of PAUF-knockout ovarian cancer cell line}

A PAUF-knockout OVCAR-5 cell line was generated using CRISPR/Cas 9 genome editing. For sgRNA, we used the CRISPR/Cas9 design tool (http://crispr.mit.edu) to select key regions of the genome for protein function and to search for sgRNA [17]. Target specificity of the sgRNA sequence to PAUF was verified by using the BLAST search in NCBI. The oligonucleotides of the sgRNA sequence (5'-

CACCGGACTACGACCATGAAATCAC-3' and 5'-AAACGTGATTTCATGGTCGTAGTCC-3'), were annealed and inserted into the BsmBI (Enzynomics) site of LentiCRISPR vector (Addgene). The mock control sequence (5'-GTTCCGCGTTACATAACTTA-3') had no other genomic matches [18]. Confirmation of transduction was performed by quantitative reverse transcription real-time polymerase chain reaction (RT-qPCR) and Western blot analysis of Cas9. The primer sequences for Cas9 RT-qPCR were 5'GGACTCCCGGATGAACTA-3' and 5'-TCGCTTCAGCTTAGGTA-3'.

\section{Proliferation assays}

Proliferation assays were performed using AlamarBlue (Invitrogen) and WST-1 (Roche). The AlamarBlue assay was carried out according to manufacturer's instructions. Briefly, cells were seeded $1 \times 10^{3}$ cells per well in 96-well plates and fluorescence was quantified using a microplate reader. The WST-1 assay was 
carried out according to manufacturer's instructions. Briefly, cells were seeded $3 \times 10^{3}$ cells per well in 96well plates and absorbance was quantified using a microplate reader. Three independent experiments were performed with duplicate samples in each. Data are presented as mean \pm SEM of three independent experiments, and representative images are shown. ${ }^{\star} P<0.05$.

\section{Migration \& invasion assays}

Migration and invasion assays were performed in Transwell plates purchased from Corning. For the invasion assay, detached cells were seeded in the upper chamber coated with Matrigel (BD Biosciences) in serum-free culture medium. The lower wells of the chamber were filled with standard medium and cells were seeded into upper chambers containing recombinant proteins or antibodies. After incubation for 20 hours at $37^{\circ} \mathrm{C}$, the cells on the upper chamber of the membrane were completely removed with a moist cotton swab. For both assays, the cells were fixed with methanol and stained, then counted and photographed by microscopy at x100 magnification. All assays were performed in duplicate and the tendency was confirmed by three independent experiments. Data are presented as mean \pm SEM of three independent experiments, and representative images are shown. ${ }^{*} P<0.05$.

\section{Adhesion assays}

Adhesion assays were performed using a 24-well plate coated with collagen type I (Sigma-Aldrich). Cells were seeded in each well and unbound cells were removed by washing twice with PBS. Cell adhesion was quantified by counting the number of stained cells. All assays were performed in triplicate and the tendencies confirmed with three independent experiments. ${ }^{*} P<0.05$.

\section{Sandwich ELISA}

For preparation of secreted PAUF, cells were cultured for three days after seeding $7 \times 10^{5}$ cells followed by concentration of culture supernatant using a Vivaspin column (Sartorius) per manufacturer's recommendation. Detection of PAUF by ELISA was as described previously [16].

\section{Ethical approval}

All animal studies were performed according to the protocol (2018-12-187, 2019-12-176) approved by Institutional Animal Care and Use Committee of Asan Institute for Life Sciences, Korea.

\section{In vivo animal experiments}

The OVCAR5 cell-derived xenograft tumor models made of female athymic nude mice (BALB/c nu/nu; 6 weeks old; Japan SLC, Hamamatsu, Japan) were used for the examination of the in vivo test. For the tumorigenesis test, OVCAR- 5 or its derivative cells were cultured and resuspended in saline.

Approximately $1 \times 10^{6}$ cells were subcutaneously implanted into the right hind leg of BALB/c nu/nu mice. For the in vivo efficacy test, the mice were implanted and allowed to rest for a period of $\sim 8$ days to allow the approximate average tumor volume to reach $100 \mathrm{~mm}^{3}$ before treatment. Groups were randomized, 
with 5 15 mice in each experimental group. A control IgG and anti-PAUF antibody at a dose of $10 \mathrm{mg} / \mathrm{kg}$ was given intravenously. Mice were treated with the dose twice a week (3-4 day intervals) for four weeks. Docetaxel (Sanofi-Aventis) was administered intravenously once for four weeks at a dose of $10 \mathrm{mg} / \mathrm{kg}$. Tumor volume was calculated by measuring 2 perpendicular diameters with a caliper and using the formula of $V=\left(a^{2} x b\right) / 2$ where, $a$ and $b$ are the short and long diameters, respectively. Mice that reached the euthanasia criteria were euthanized by carbon dioxide $\left(\mathrm{CO}_{2}\right)$ inhalation. Body weight was measured twice a week (3-4 day intervals) on the day of tumor measurement. Data for tumor and body weight were plotted to indicate the tumor growth curve and body weight change. Survival was analyzed by the KaplanMeier method, and comparisons between groups were analyzed by the Wilcoxon and log-rank tests.

\section{Statistical analysis}

Data generated from at least three experiments performed in triplicate were analyzed using the two-tailed Student's t-test. Results were considered significant when $P<0.05$.

\section{Results}

\section{PAUF enhances the migration and invasion of human serous ovarian cancer cells in vitro}

To determine the endogenous levels of PAUF expression in various human serous ovarian adenocarcinoma cell lines, cells were cultured and a sandwich ELISA of culture supernatants was conducted. Levels of PAUF were highest in OVCAR- 5 cells ( 19 ng per $1 \times 10^{6}$ cells) while HeyA8, A2780, SK-OV-3, and OVCAR-8 cells showed modest expression (Fig. 1a). PAUF expression in OVCAR-5 was higher when compared with CFPAC-1, the pancreatic cancer cell line previously shown to express PAUF at high levels [19] (Fig. 1b). Next, the potential impact of PAUF on the motility and invasiveness of serous ovarian cancer cell lines was characterized. The three cell lines with a modest PAUF expression-HeyA8, SK-OV-3, and OVCAR-8-were treated with recombinant PAUF (rPAUF). As shown in Fig. 1c-f, migration, invasion, and adhesion activities increased significantly in the treated cells when compared to the control cells (i.e., no PAUF). These results are consistent with our previous findings in pancreatic cancer cell lines $[8,10]$. However, the treatment did not show significant changes in the motility and invasiveness of the OVCAR-5 cell line, which expresses PAUF at a high level. Treatment of OVCAR-8 cells with rPAUF activated intracellular signaling pathways involving ERK, Src, and AKT (Fig. 1g and Fig. S1), implying that PAUF may exert similar functions in both ovarian and pancreatic cancer cells [8-10]. These results suggest that PAUF may function as a factor that affects the metastasis in serous ovarian adenocarcinoma.

\section{Reduced PAUF activity diminishes migration, invasion, and proliferation of serous ovarian cancer cells in vitro}

We explored the effects of an antibody that neutralizes PAUF function. The anti-PAUF antibody significantly inhibited the migration and invasion of OVCAR-5 cells (Fig. 2). The inhibitory activity of the anti-PAUF antibody was slightly less in cell lines with lower PAUF expression (i.e., HeyA8, SK-OV-3, OVCAR-8). To determine whether cancer-related cellular activities could be attenuated by reducing the 
level of PAUF, we established a stable PAUF-knockout OVCAR-5 cells line (OVCAR-5_PAUF K/O as described in the Materials and Methods). Successful transduction was confirmed by RT-qPCR and immunoblot analysis (Fig. 3a, b and Fig. S2). OVCAR-5_Mock cells were established as a negative control. As determined by sandwich ELISA, the level of PAUF expression in the OVCAR-5_PAUF K/O cells was reduced by more than $90 \%$ compared to the OVCAR-5_Mock cells (Fig. $3 \mathrm{C}$ ). PAUF K/O cells exhibited significantly lower migratory and invasion capabilities when compared to the control cells (Fig. 3d, e). PAUF K/O cells also showed a decrease in proliferative capacity (Fig. 3f). Treatment of PAUF K/O cells with rPAUF recovered the motility, invasion, and proliferation abilities (Fig. $3 \mathrm{~g}$ ). Therefore, both functional inhibition and expressional knockout of PAUF resulted in lowered cancer cell activities, suggesting that PAUF may be used for a targeted anti-cancer therapy to treat serous ovarian adenocarcinoma.

\section{PAUF-knockout delayed in vivo xenograft tumor growth}

In vivo relevance of the in vitro results was determined by evaluating the tumorigenesis and tumor growth rate in ovarian xenograft tumor models. Mice were subcutaneously implanted with OVCAR-5_PAUF K/O or OVCAR-5_Mock cells and tumor growth curves were plotted using tumor volumes measured twice a week for 46 days (Fig. 4). Individual and average tumor growth curves are shown in Fig. 4a, b, respectively. Overall, tumor growth in the OVCAR-5_PAUF K/O group was slower when compared with the OVCAR5_Mock group. It took a mean of 32 days for tumors in the OVCAR-5_Mock group to reach $500 \mathrm{~mm}^{3}$ compared with 36 days in the OVCAR-5_PAUF K/O group, indicating delayed tumor growth by PAUFknockout. At the endpoint of the experiment, the tumor growth inhibition (TGI) percentages were calculated. On day 46, the average tumor volumes of OVCAR-5_PAUF K/O and OVCAR-5_ Mock groups were $1,200 \mathrm{~mm}^{3}$ and $1,800 \mathrm{~mm}^{3}$, respectively, correlating to a TGI of $32.92 \%$ from PAUF-knockout (Fig. 4c). The largest and smallest volumes of OVCAR-5_PAUF K/O group were $1,300 \mathrm{~mm}^{3}$ and $800 \mathrm{~mm}^{3}$, while those volumes of OVCAR-5_Mock group were $2,000 \mathrm{~mm}^{3}$ and $1,500 \mathrm{~mm}^{3}$. These results strongly suggested that the PAUF expression was involved in ovarian tumor progression in vivo and could be a therapeutic target for human ovarian cancer treatment.

\section{An anti-PAUF antibody demonstrated therapeutic efficacy in an ovarian cancer xenograft mouse model}

We tested whether an anti-PAUF antibody is an effective treatment for human serous ovarian cancer. Mice bearing OVCAR-5-derived subcutaneous xenograft tumors were randomly divided into two groups and treated with $10 \mathrm{mg} / \mathrm{kg}$ anti-PAUF antibody or control IgG intravenously twice a week (Fig. 5a-d). The tumor growth rate and distribution of tumor volumes between IgG-treated and anti-PAUF antibody-treated groups were analyzed; average tumor growth curves are shown in Fig. $5 \mathrm{a}$. The results indicate that the anti-PAUF antibody tested here is capable of reducing the growth of OVCAR- 5 xenograft tumors in a mouse model. As shown in Fig. 5b, on day 23, the average tumor volumes of the anti-PAUF antibodytreated and control IgG-treated groups were $1,400 \mathrm{~mm}^{3}$ and $1,800 \mathrm{~mm}^{3}$, respectively, reflecting a $27 \% \mathrm{TGI}$ effect of treatment with an anti-PAUF antibody. A Kaplan-Meier analysis indicated that the survival rate of the anti-PAUF antibody-treated group increased $28.6 \%$ compared to that of the control IgG-treated group (Fig. 5c). There was no difference in body weight between the two groups (Fig. 5d). To test the 
combination effect of the antibody with docetaxel, the tumor growth rate and distribution of tumor volumes between IgG, anti-PAUF antibody, docetaxel, and combination-groups were analyzed; average tumor growth curves are shown in Fig. $5 \mathrm{e}$. As shown in Fig. 5f, on day 31 the average tumor volumes of the control IgG, anti-PAUF antibody, docetaxel, and combination treated groups were $2,600 \mathrm{~mm}^{3}, 2,200$ $\mathrm{mm}^{3}, 1,700 \mathrm{~mm}^{3}$, and $1,600 \mathrm{~mm}^{3}$, respectively. In the current experiment, a Kaplan-Meier analysis indicated that while the anti-PAUF antibody showed a $17 \%$ improvement of the median survival rate when compared with control IgG, docetaxel alone showed $24 \%$ compared with control IgG. Furthermore, the combination of docetaxel and anti-PAUF antibody exhibited a significantly improved median survival rate of $29 \%$ (Fig. 5g). There was no difference in body weight among the test groups (Fig. 5h). Our results clearly demonstrate that the anti-PAUF antibody reduced tumor growth and improved survival in OVCAR5-derived xenograft tumor models, suggesting that PAUF could be targeted by therapeutic antibodies for the treatment of human ovarian serous carcinoma.

\section{Discussion}

The current study provides evidence that targeting PAUF may serve as an effective modality for the treatment of serous ovarian adenocarcinoma, which is the first report of these finding to date. Here, lines of evidence are presented for the identification of PAUF as a potential therapeutic target for serous ovarian cancer. First, variations in the levels of PAUF, achieved through either knockout or exogenous treatment approaches, led to changes in tumor related-phenotypes (i.e., migration, invasion, adhesion, proliferation) of serous ovarian cancer cells. Second, the intracellular signaling pathways activated by PAUF in serous ovarian cancer cells were similar to those observed in pancreatic cancer cells $[8,10]$. Third, PAUF-knockout led to delayed tumor growth in a xenograft mouse model with the serous ovarian cancer cell line OVCAR-5. Finally, treatment of mice bearing OVCAR-5-derived xenograft tumors with a PAUF-neutralizing antibody resulted in retarded tumor growth and improved survival.

Ultrasonography and cancer antigen 125 (CA125) are standard methods for the detection of ovarian cancer [20]. However, early diagnosis of ovarian cancer is limited as diagnosis can be inaccurate with ultrasonography, and CA125, which is also detected in benign gynecological diseases and other tumors, rises only in $50 \%$ to $60 \%$ in early-stage ovarian cancer patients [21]. As a result, roughly $70 \%$ of ovarian cancer patients are diagnosed with an advanced stage of the disease [1, 2]. Therefore, research to identify new tumor biomarkers that can supplement or replace CA125 are needed. Two recent publications have highlighted the clinical importance of PAUF in ovarian cancer, suggesting PAUF as a prognostic factor in epithelial ovarian cancer $[15,16]$. PAUF is highly expressed in epithelial ovarian adenocarcinoma and associated with tumor grade and chemoresistance[15, 16]. PAUF expression correlates with poor prognosis for both progression-free survival and overall survival. Here, we show that PAUF is a malignant factor in the progression of serous ovarian cancer in vitro and in vivo. Taken together, we propose PAUF is a potential novel biomarker for serous ovarian cancer.

Current treatment for ovarian cancer includes surgery and platinum-based chemotherapy [4], however, the majority of patients with advanced stages develop recurrent disease and resistance to chemotherapy [5, 
6]. Many targeted treatment approaches, including biological drugs, are under development with limited clinical benefits reported to date [22]. Targeting PAUF has been shown to be effective in inhibiting pancreatic tumor growth in xenograft mouse models. A PAUF-specific aptamer or suppression of PAUF expression inhibits the progression of the tumor growth $[23,24]$ and enhances chemotherapy sensitivity $[25,26]$. Furthermore, a PAUF-neutralizing antibody reduced tumor growth and distant metastasis [19]. Here we show that PAUF-knockout or anti-PAUF antibody limits ovarian tumor growth in xenograft mouse models, implying that targeting PAUF could be an effective therapeutic modality to treat serous ovarian adenocarcinoma as well as pancreatic ductal adenocarcinoma. Although the pathogenic differences between pancreatic and ovarian tumors are not yet clear [27], we suggest that PAUF overexpression is a common risk factor for these diseases.

Further studies should include developing a non-invasive method to detect PAUF for diagnosis and prognosis of ovarian cancer, validating the efficacy of the anti-PAUF antibody in models reflecting the heterogeneity of patients, and evaluating of the therapeutic antibody in combination with chemical drugs.

\section{Declarations}

\section{Author Contributions}

Yeon Jeong Kim and Sang Seok Koh designed the study. Yeon Jeong Kim, Jin Park, Hyeon Hee Jeong and Ji Eun Baek performed the experiments. Seung-Mo Hong, Seong-Yun Jeong and Sang Seok Koh analyzed the data. The first draft of the manuscript was written by Yeon Jeong Kim, Jin Park and Sang Seok Koh and all authors commented on previous versions of the manuscript. All authors read and approved the final manuscript.

\section{Funding Information}

This study was supported by grant from the Korean Health Technology R\&D Project through the Korea Health Industry Development Institute (KHIDI), funded by the Ministry for Health \& Welfare, Republic of Korea (HA15C0002).

\section{Data Availability}

All data generated or analyzed during this study are included in this published article and its supplementary information files.

\section{Compliance with Ethical Standards}

\section{Conflict of Interest}

The authors declare that they have no conflict of interest.

\section{Ethical Approval}


All animal studies were performed according to the protocol (2018-12-187, 2019-12-176) approved by Institutional Animal Care and Use Committee of Asan Institute for Life Sciences, Korea.

\section{References}

1. Siegel RL, Miller KD, Jemal A. Cancer statistics 2018. CA Cancer J Clin. 2018;68(1):7-30. https://doi.org/10.3322/caac.21442.

2. Lheureux S, Braunstein M, Oza AM. Epithelial ovarian cancer: Evolution of management in the era of precision medicine. CA Cancer J Clin. 2019;69(4):280-304. https://doi.org/10.3322/caac.21559.

3. Devouassoux-Shisheboran M, Genestie C. Pathobiology of ovarian carcinomas. Chin J Cancer. 2015;34(1):50-5. https://doi.org/10.5732/cjc.014.10273.

4. Raja FA, Chopra N, Ledermann JA. Optimal first-line treatment in ovarian cancer. Ann Oncol. 2012;23:x118-27. https://doi.org/10.1093/annonc/mds315.

5. Dao F, Schlappe BA, Tseng J, Lester J, Nick AM, Lutgendorf SK, McMeekin S, Coleman RL, Moore LN, Karlan BY, Sood AK, Levine DA. Characteristics of 10-year survivors of high-grade serous ovarian carcinoma. Gynecol Oncol. 2016;141(2):260-3. https://doi.org/10.1016/j.ygyno.2016.03.010.

6. Christie EL, Bowtell DDL. Acquired chemotherapy resistance in ovarian cancer. Ann Oncol. 2017;28(suppl_8):viii13-5. https://doi.org/10.1093/annonc/mdx446.

7. Kim SA, Lee Y, Jung DE, Park KH, Park JY, Gang J, Jeon J, Park SB, Kim EC, Lee YG, Liu B, Zeng Q, Yeramilli W, Lee S, Koh S, Song SS SY. Pancreatic adenocarcinoma up-regulated factor (PAUF), a novel up-regulated secretory protein in pancreatic ductal adenocarcinoma. Cancer Sci. 2009;100(5):828-36. https://doi.org/10.1111/j.1349-7006.2009.01106.x.

8. Lee Y, Kim SJ, Park HD, Park EH, Huang SM, Jeon SB, Kim JM, Lim DS, Koh SS. PAUF functions in the metastasis of human pancreatic cancer cells and upregulates CXCR4 expression. Oncogene. 2010;29(1):56-67. https://doi.org/10.1038/onc.2009.298.

9. Cho IR, Koh SS, Min HJ, Kim SJ, Lee Y, Park EH, Ratakorn S, Jhun BH, Oh S, Johnston RN, Chung YH. Pancreatic adenocarcinoma up-regulated factor (PAUF) enhances the expression of beta-catenin, leading to a rapid proliferation of pancreatic cells. Exp Mol Med. 2011;43(2):82-90. https://doi.org/10.3858/emm.2011.43.2.010.

10. Lee YS, Kim SJ, Min HJ, Jo JY, Park EH, Koh SS. PAUF promotes adhesiveness of pancreatic cancer cells by modulating focal adhesion kinase. Exp Mol Med. 2011;43(5):291-7. https://doi.org/10.3858/emm.2011.43.5.030.

11. Cho IR, Koh SS, Malilas W, Srisuttee R, Moon J, Choi YW, Horio Y, Oh S, Chung YH. SIRT1 inhibits proliferation of pancreatic cancer cells expressing pancreatic adenocarcinoma up-regulated factor (PAUF), a novel oncogene, by suppression of $\beta$-catenin. Biochem Biophys Res Commun. 2012;423(2):270-5. https://doi.org/10.1016/j.bbrc.2012.05.107.

12. Kim SJ, Lee Y, Kim NY, Hwang Y, Hwang B, Min JK, Koh SS. Pancreatic adenocarcinoma upregulated factor, a novel endothelial activator, promotes angiogenesis and vascular permeability. Oncogene. 
2013;32(31):3638-47. https://doi.org/10.1038/onc.2012.366.

13. Park HD, Lee Y, Oh YK, Jung JG, Park YW, Myung K, Kim KH, Koh SS, Lim DS. Pancreatic adenocarcinoma upregulated factor promotes metastasis by regulating TLR/CXCR4 activation. Oncogene. 2011;30(2):201-11. https://doi.org/10.1038/onc.2010.401.

14. Song J, Lee J, Kim J, Jo S, Kim YJ, Baek JE, Kwon ES, Lee KP, Yang S, Kwon KS, Kim DU, Kang TH, Park YY, Chang S, Cho HJ, Kim SC, Koh SS, Kim S. Pancreatic adenocarcinoma up-regulated factor (PAUF) enhances the accumulation and functional activity of myeloid-derived suppressor cells (MDSCs) in pancreatic cancer. Oncotarget. 2016;7(32):51840-53.

https://doi.org/10.18632/oncotarget.10123.

15. Kim SK, Song SY, Kim S, Cho NH, Yim GW, Kim SW, Kim YT, Nam EJ. Association of pancreatic adenocarcinoma up-regulated factor expression in ovarian mucinous adenocarcinoma with poor prognosis. Int J Clin Exp Pathol. 2014;7(8):5103-10. eCollection 2014.

16. Choi CH, Kang TH, Song JS, Kim YS, Chung EJ, Ylaya K, Kim S, Koh SS, Chung JY, Kim JH, Hewitt SM. Elevated expression of pancreatic adenocarcinoma upregulated factor (PAUF) is associated with poor prognosis and chemoresistance in epithelial ovarian cancer. Sci Rep. 2018;8(1):12161. https://doi.org/10.1038/s41598-018-30582-8.

17. Shalem O, Sanjana NE, Hartenian E, Shi X, Scott DA, Mikkelson T, Heckl D, Ebert BL, Root DE, Doench JG, Zhang F. Genome-scale CRISPR-Cas9 knockout screening in human cells. Science. 2014;343(6166):84-7. https://doi.org/10.1126/science.1247005.

18. Kearns NA, Genga RM, Enuameh MS, Garber M, Wolfe SA, Maehr R. Cas9 effector-mediated regulation of transcription and differentiation in human pluripotent stem cells. Development. 2014;141(1):219-23. https://doi.org/10.1242/dev.103341.

19. Kim SJ, Chang S, Lee Y, Kim NY, Hwang Y, Min HJ, Yoo KS, Park EH, Kim S, Chung YH, Park YW, Koh SS. A PAUF-neutralizing antibody targets both carcinoma and endothelial cells to impede pancreatic tumor progression and metastasis. Biochem Biophys Res Commun. 2014;454(1):144-50. https://doi.org/10.1016/j.bbrc.2014.10.056.

20. Altman DG, Riley RD. Primer: an evidence-based approach to prognostic markers. Nat Clin Pract Oncol. 2015;2(9):466-72. https://doi.org/10.1038/ncponc0287.

21. Cohen JG, White M, Cruz A, Farias-Eisner R. In 2014, can we do better than CA125 in the early detection of ovarian cancer? World J Biol Chem. 2014;5(3):286-300. https://doi.org/10.4331/wjbc.v5.i3.286.

22. Cortez AJ, Tudrej P, Kujawa KA, Lisowska KM. Advances in ovarian cancer therapy. Cancer Chemother Pharmacol. 2018;81(1):17-38. https://doi.org/10.1007/s00280-017-3501-8.

23. Kim YH, Sung HJ, Kim S, Kim EO, Lee JW, Moon JY, Choi K, Jung JE, Lee Y, Koh SS, Rhee SG, Heo K, $\mathrm{Kim} \mathrm{IH}$. An RNA aptamer that specifically binds pancreatic adenocarcinoma up-regulated factor inhibits migration and growth of pancreatic cancer cells. Cancer Lett. 2011;313(1):76-83. https://doi.org/10.1016/j.canlet.2011.08.027. 
24. Kim YH, Moon JY, Kim EO, Lee SJ, Kang SH, Kim SK, Heo K, Lee Y, Kim H, Kim KT, Kim D, Song MS, Lee SW, Lee Y, Koh SS, Kim IH. Efficient targeting and tumor retardation effect of pancreatic adenocarcinoma up-regulated factor (PAUF)-specific RNA replacement in pancreatic cancer mouse model. Cancer Lett. 2014;344(2):223-31. https://doi.org/10.1016/j.canlet.2013.10.028.

25. Gao CC, Xu XL, Li F, Gong BG, Liu S, Cui YQ, Sun HC, Xu PY, Zheng YM, Jiang H. Silencing pancreatic adenocarcinoma upregulated factor (PAUF) increases the sensitivity of pancreatic cancer cells to gemcitabine. Tumour Biol. 2016;37(6):7555-64. https://doi.org/10.1007/s13277-015-4641-2.

26. Cho JH, Kim SA, Park SB, Kim HM, Song SY. Suppression of pancreatic adenocarcinoma upregulated factor (PAUF) increases the sensitivity of pancreatic cancer to gemcitabine and $5 \mathrm{FU}$, and inhibits the formation of pancreatic cancer stem like cells. Oncotarget. 2017;8(44):76398-407. https://doi.org/10.18632/oncotarget.19458.

27. Kim S, Han Y, Kim SI, Kim HS, Kim SJ, Song YS. Tumor evolution and chemoresistance in ovarian cancer. NPJ Precis Oncol. 2018;2:20. https://doi.org/10.1038/s41698-018-0063-0.

\section{Figures}

Fig. 1

Kim et al

a

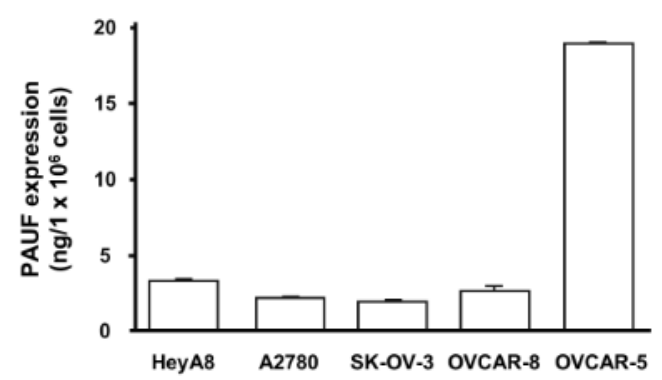

b

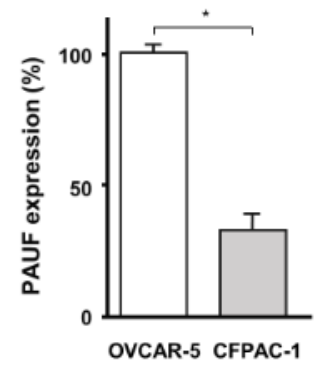

c

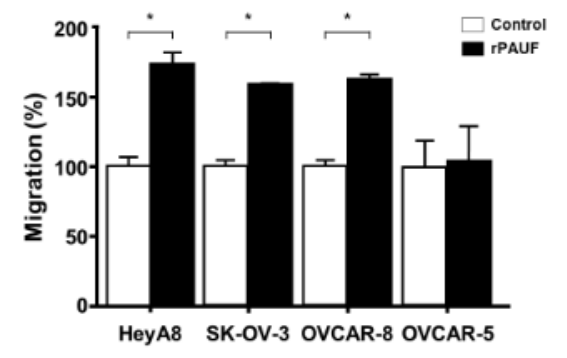

f

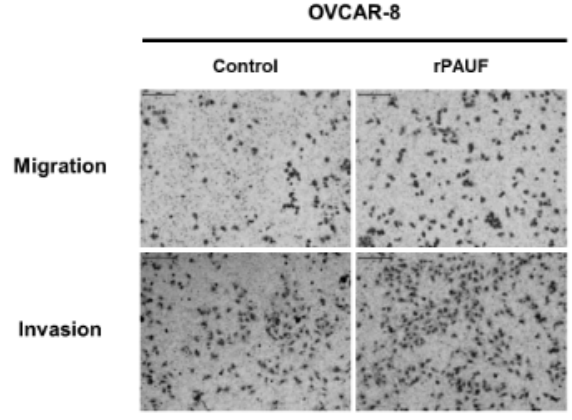

d

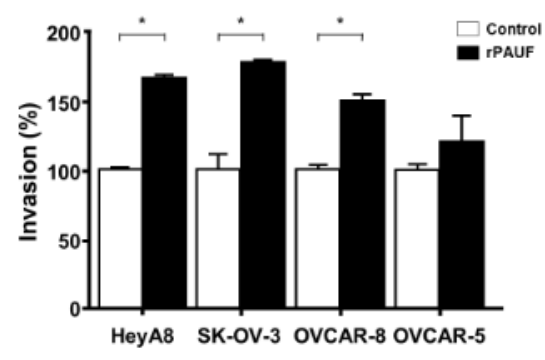

e

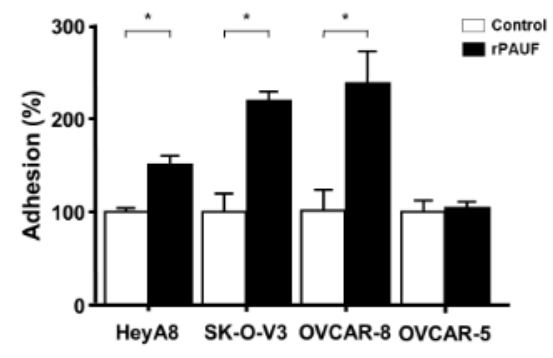

g OVCAR-8

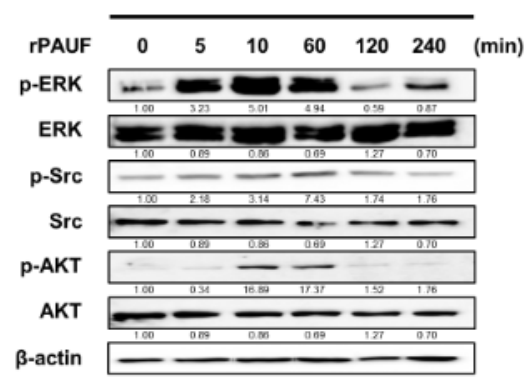


Figure 1

PAUF enhances the migration and invasion of human serous ovarian cancer cells. (a) PAUF expression in human serous ovarian cancer cell lines was measured by ELISA. (b) Comparison of PAUF expression in OVCAR-5 and CFPAC-1. (c-e) Serous ovarian adenocarcinoma cells were incubated in the presence of rPAUF $(0.5 \mu \mathrm{g} / \mathrm{ml})$ or PBS as control. Cell migration (c), invasion (d), and adhesion (e) were assessed as described in the Materials and Methods. (f) Representative images of OVCAR-8 cell migration and invasion of the chamber membrane. $(\mathrm{g})$ Intracellular signaling pathways in serous ovarian adenocarcinoma cells. OVCAR-8 cell was serum-starved for 16 hours and treated with $0.1 \mu \mathrm{g} / \mathrm{ml}$ of rPAUF for $0,5,10,60,120$, and 240 min. Cell lysates were prepared and used for Western blot analysis with respective $\mathrm{p}$-ERK, ERK, Src, p-Src, AKT, or p-AKT antibody. $\beta$-actin was used as a loading control. Three independent experiments were performed with duplicate samples in each. Data are presented as mean \pm SEM of three independent experiments, and representative images are shown. ${ }^{*} \mathrm{P}<0.05$.

Fig. 2

Kim et al

a

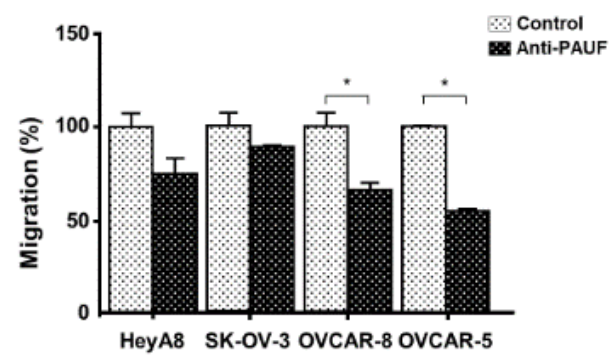

c

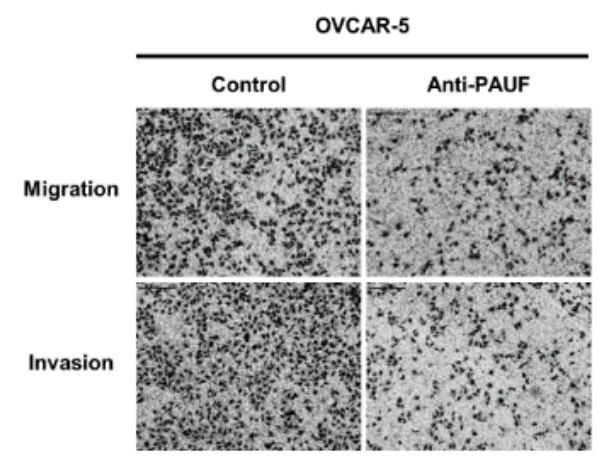

b

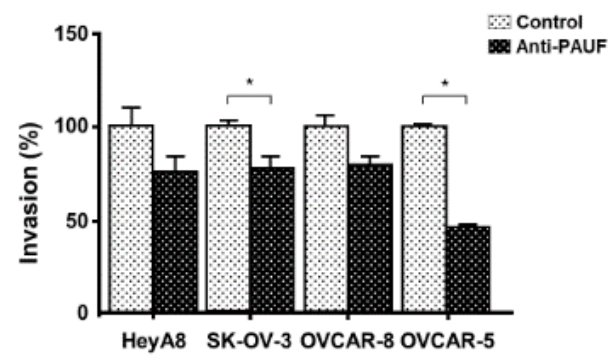

\section{Figure 2}

Anti-PAUF antibody inhibits the migration and invasion of human serous ovarian cancer cells. Cells were incubated with an anti-PAUF antibody $(10 \mu \mathrm{g} / \mathrm{ml})$ or control $\mathrm{lgG}(10 \mu \mathrm{g} / \mathrm{ml})$. Cell migration (a) and invasion (b) were assessed as described in Figure 1. (c) Representative images of OVCAR-5 cell migration 
and invasion of the chamber membrane. Three independent experiments were performed with duplicate samples in each. Data are presented as mean \pm SEM of three independent experiments, and representative images are shown. * $\mathrm{P}<0.05$.

Fig. 3

Kim et al

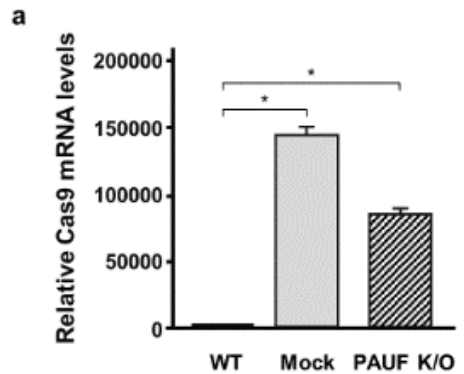

b

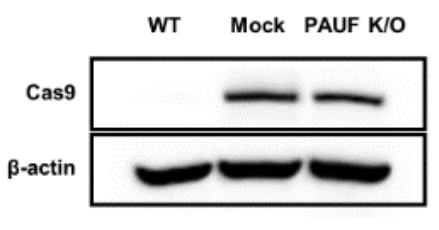

c

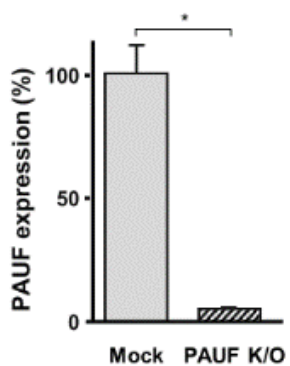

d

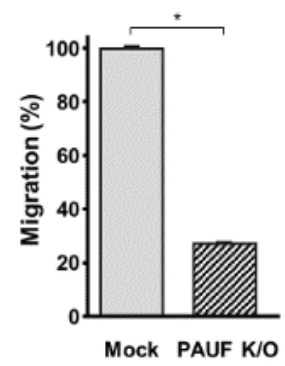

g e

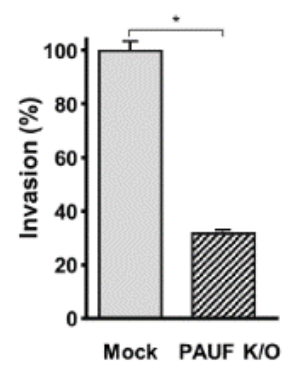

f

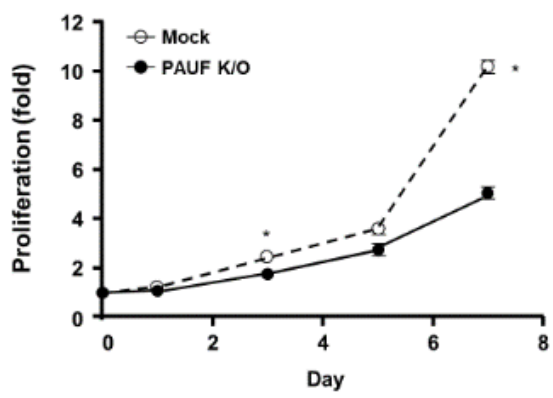

PAUF K/O
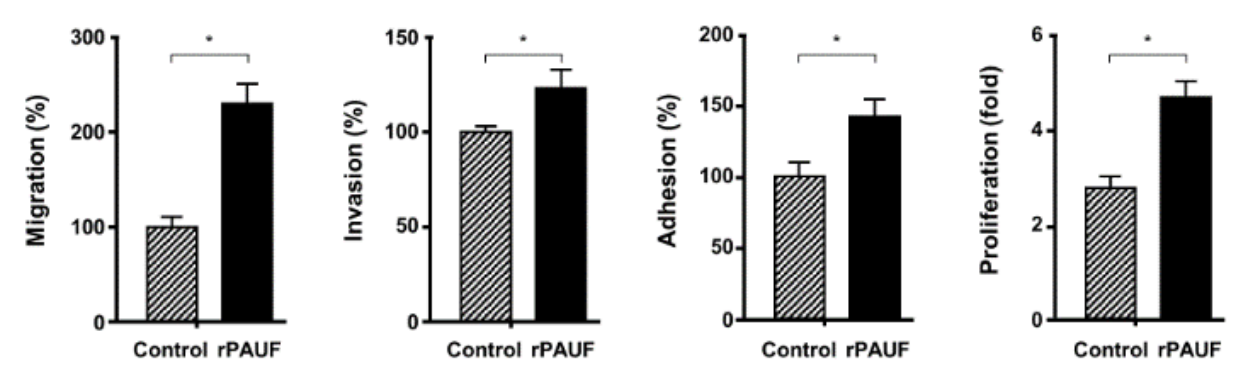

\section{Figure 3}

Migration, invasion, and proliferation of OVCAR-5 cells are decreased by PAUF-knockout. The PAUFknockout OVCAR-5 cell line was generated using CRISPR/Cas9 genome editing. Confirmation of transduction was performed by RT-qPCR (a) and Western blot analysis (b) of Cas9. Relative expression levels were determined by normalizing the expression level of each target to GAPDH. $\beta$-actin was used as a loading control. (c) PAUF levels were determined using sandwich ELISA of culture supernatants of OVCAR-5_PAUF K/O and OVCAR-5_Mock cells. (d-f) Motility and proliferation of OVCAR-5_PAUF K/O and OVCAR-5_Mock cells were determined. Cell migration (d) and invasion (e) were assayed as described in Figure 1. (f) Cell proliferation assays were performed using a proliferation indicator, and fluorescence was quantified using a microplate reader. Three independent experiments were performed with duplicate samples in each. (g) Cell migration, invasion, adhesion, and proliferation of OVCAR-5_PAUF K/O cells were measured in presence of rPAUF $(0.5 \mu \mathrm{g} / \mathrm{ml})$ or PBS as control. Cell proliferation was measured using WST-1 on day 7. Data are presented as mean \pm SEM and representative images are shown. ${ }^{*} \mathrm{P}<0.05$. 
Fig. 4

Kim et al

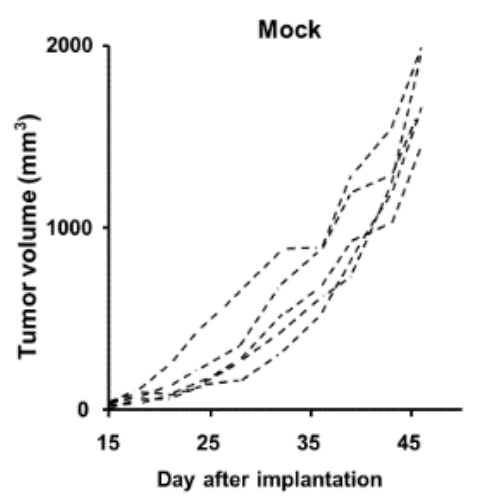

b

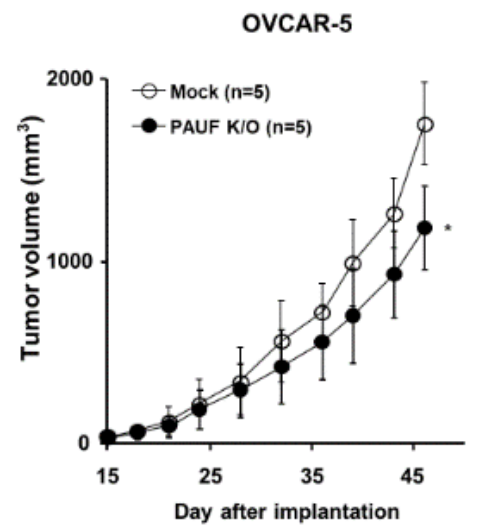

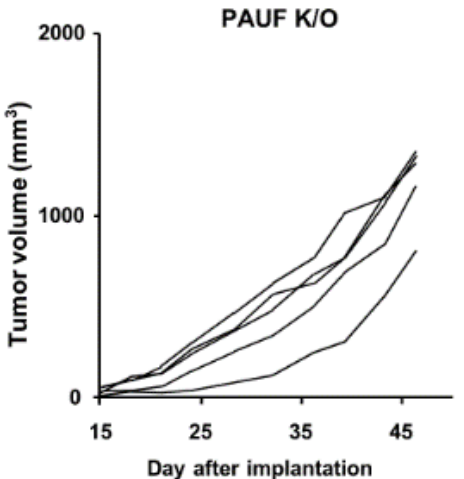

c

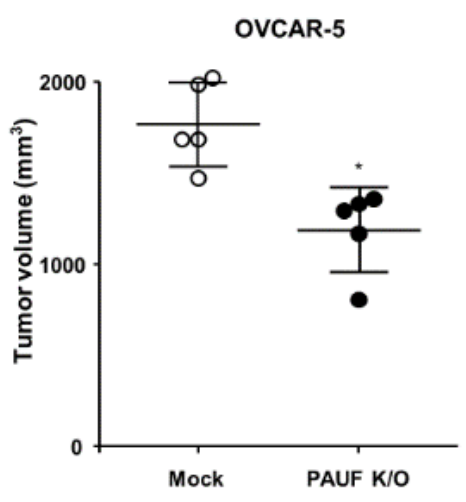

\section{Figure 4}

PAUF-knockout delays tumor growth in vivo. OVCAR-5_PAUF K/O and OVCAR-5_Mock cells were subcutaneously implanted into the right hind leg of BALB/c nu/nu mice ( $n=5 /$ group). Tumor volumes were measured twice a week (every 3-4 days) and plotted individually (a) and using averages (b) throughout the experimental period. (c) The distribution of tumor volumes in each group at the endpoint of the experiment on day 46 . Tumor volumes measured on day 46 were plotted individually. ${ }^{*} \mathrm{P}<0.05$. 


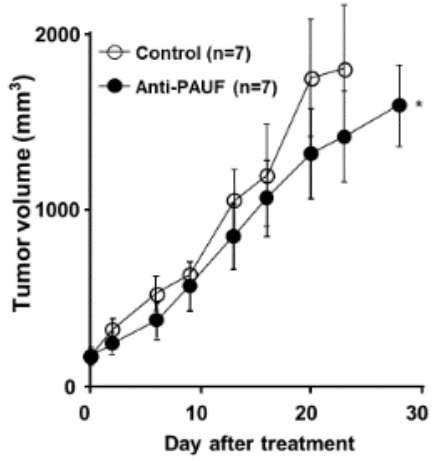

e

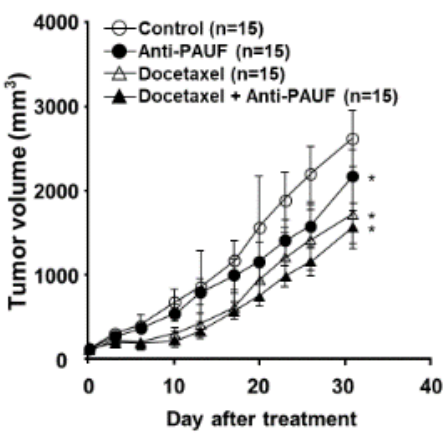

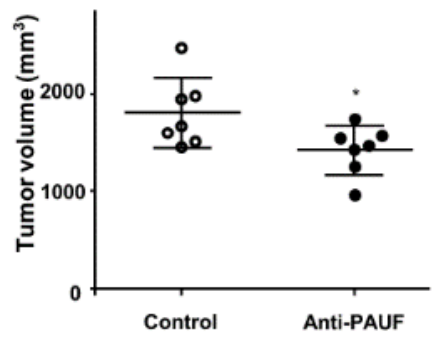

f

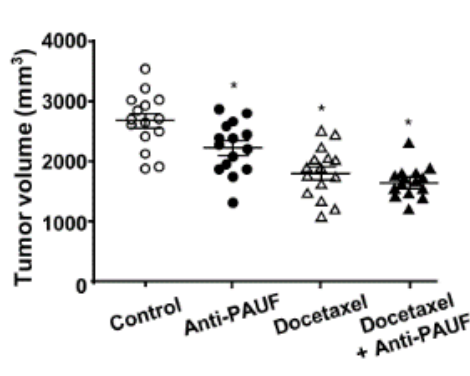

g
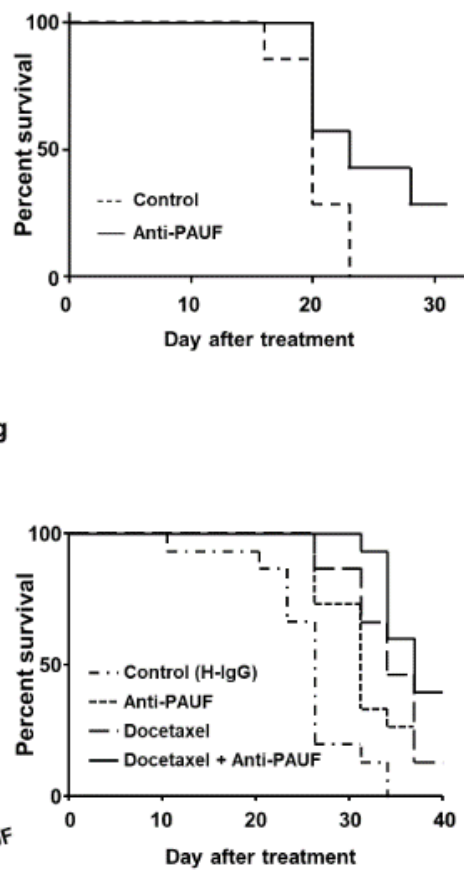

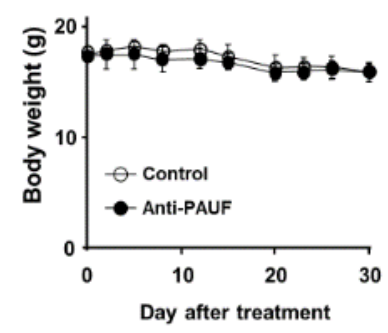

h

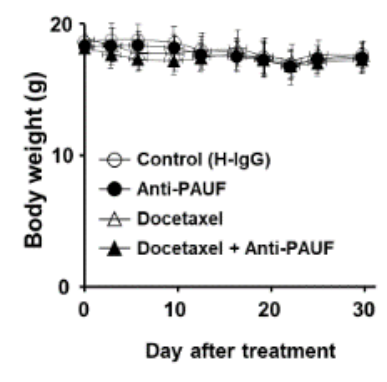

Figure 5

Anti-PAUF antibody displayed a therapeutic efficacy in ovarian cancer xenograft mouse model. (a) Tumor growth curves of OVCAR-5 xenograft mouse models treated with an anti-PAUF antibody $(10 \mathrm{mg} / \mathrm{kg})$ or control lgG (10 mg/kg). The OVCAR-5 cells were subcutaneously implanted into the right hind leg of BALB/c nu/nu mice ( $n=7 / g r o u p)$. Mice were randomized into experimental groups once tumors reached approximately $100 \mathrm{~mm}$. Antibodies were administered intravenously twice a week (3-4 days intervals) for four weeks and plotted as averages throughout the experimental period. (b) The distribution of tumor volumes in groups at the endpoint of the experiment on day 23. (c) Kaplan-Meier curves for survival. Survival time was defined as the number of days until tumor volume reached 1,500 mm3. (d) The body weight of each mouse was measured every 3-4 days throughout the experimental period. (e) Tumor growth curves of OVCAR-5 xenograft mouse models treated with an anti-PAUF antibody (10 mg/kg), docetaxel $(10 \mathrm{mg} / \mathrm{kg})$, or a combination of both. Control lgG (10 mg/kg) was used as a negative control. The OVCAR- 5 cells were subcutaneously implanted into the right hind leg of BALB/c nu/nu mice ( $n=15 /$ group). Antibodies were administered intravenously twice a week (3-4 days intervals) for four weeks and docetaxel was administered once during the experimental period. Tumor volumes were measured twice a week (every 3-4 days) and plotted using averages throughout the experimental period. 
(f) The distribution of tumor volumes in groups at the endpoint of the experiment on day 31. (g) KaplanMeier curves for survival. Survival time was defined as the number of days until the tumor volume reached 2,000 mm3. (h) The body weight of each mouse was measured every 3-4 days throughout the experimental period. ${ }^{*} \mathrm{P}<0.05$.

\section{Supplementary Files}

This is a list of supplementary files associated with this preprint. Click to download.

- KimSupplementarylnformation.pdf 